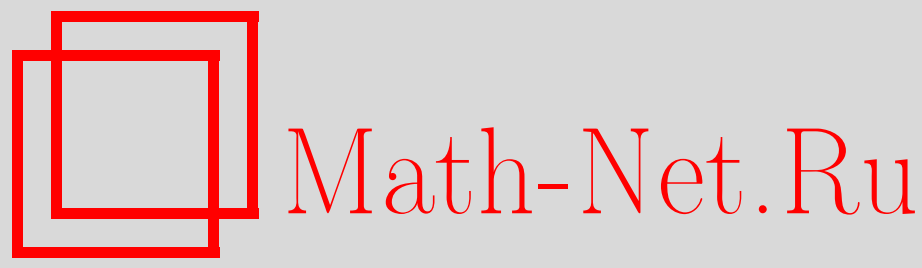

В. Ю. Новокшенов, Аппроксимации Паде для трансцендентов Пенлеве I и II, TMФ, 2009, том 159, номер 3, 515526

DOI: https://doi.org/10.4213/tmf6369

Использование Общероссийского математического портала Math-Net.Ru подразумевает, что вы прочитали и согласны с пользовательским соглашением http: //www.mathnet.ru/rus/agreement

Параметры загрузки:

IP : 54.224 .187 .69

26 апреля 2023 г., 13:27:47

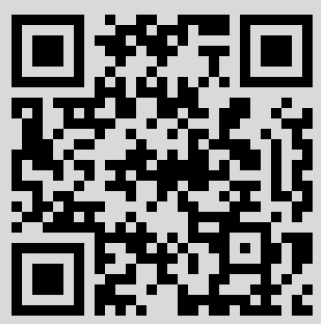




\title{
ФИЗИКА
}

Том 159, № 3

июнь, 2009

\section{АППРОКСИМАЦИИ ПАДЕ ДЛЯ ТРАНСЦЕНДЕНТОВ ПЕНЛЕВЕ I И ІІ}

\begin{abstract}
Для нахождения аппроксимации Паде решений уравнений Пенлеве I и II использован вариант алгоритма Фейра-Льюка. Найдены распределения полюсов хорошо известных решений Абловица-Сегура и Хастингса-Маклеода уравнения Пенлеве II. Показано, что трижды усеченное решение Бутру уравнения Пенлеве I имеет полюсы только в критическом секторе комплексной плоскости. Данный алгоритм позволяет проверить другие аналитические свойства трансцендентов Пенлеве, такие как асимптотики на бесконечности в комплексной плоскости.
\end{abstract}

Ключевые слова: уравнение Пенлеве, мероморфное решение, распределение полюсов, аппроксимация Паде, непрерывная дробь, задача Римана-Гильберта.

\section{1. ВВЕДЕНИЕ}

Со времени работ Пенлеве, доказавшего мероморфность всех решений уравнения Пенлеве I (ПI)

$$
u^{\prime \prime}=6 u^{2}-z, \quad z \in \mathbb{C},
$$

исследование распределения полюсов стало одной из задач аналитической теории нелинейных обыкновенных дифференциальных уравнений (ОДУ) (см. [1]). Бутру [2] впервые исследовал трансценденты ПІ в общем положении и установил основные свойства их полюсов (см. раздел 3). В отличие от общих оценок в духе теории Неванлинны, поведение трансцендентов Пенлеве более регулярно на бесконечности или вблизи существенных сингулярностей. Это доказано с помощью аппроксимаций эллиптическими функциями, приближающих решения в подходящих секторах комплексной плоскости (см. раздел 4).

Итерации рациональных решений уравнений Пенлеве были открыты достаточно давно. Регулярный метод порождения этих решений для уравнений ПII-ПVI основан на преобразованиях Беклунда ([1], глава 6). Они использовались в ряде работ (см., например, [3], [4]) для построения семейств рациональных решений. Используя формулы Воробьева-Яблонского и многочлены Окамото, Кларксон построил

* Институт математики, Уфимский научный центр РАН, Уфа, Россия.

E-mail: novik53@mail.ru 
распределения полюсов для уравнений ПII-ПV с большими коэффициентами [5]. Однако при фиксированном наборе коэффициентов этот метод не дает результата, поскольку после каждого преобразования Беклунда коэффициенты сдвигаются. Более того, данный метод бесполезен в применении к уравнению ПІ, где нет свободных коэффициентов и нет рациональных решений.

Другие подходы были предложены в работах [6] и [7]. В работе [6] для автомодельного решения нелинейного уравнения Шредингера полюсы в уравнении ПIV рассматриваются как кулоновский газ в параболическом потенциале. Однако для большого числа частиц соответствующая вариационная задача не очень проста. В работе [7] на основе нелинейного ОДУ четвертого порядка был найден аналог многочленов Окамото для уравнения ПІ.

Предложенные ниже аппроксимации Паде хорошо подходят для уравнений Пенлеве по следующим причинам. Во-первых, они учитывают инвариантность относительно преобразований Мёбиуса в классе нелинейных ОДУ, включающем уравнения Пенлеве. Во-вторых, они сходятся и дают сколь угодно хорошие аппроксимации, поскольку все решения являются мероморфными (см. раздел 2). Численные эксперименты, проведенные с использованием этого метода, позволили установить новые факты и привели к формулировке гипотез, которые необходимо доказать аналитически.

\section{2. АЛГОРИТМ ФЕЙРА-ЛЬЮКА}

Поскольку все решения уравнения ПІ (1) являются мероморфными функциями, естественно применить аппроксимацию Паде, по крайней мере, в компактных областях. Если $z=0$ не является полюсом функции $v(z)$, то можно определить диагональную аппроксимачию Паде

$$
v(z)=\sum_{k=0}^{\infty} c_{k} z^{k} \approx \frac{P_{N}(z)}{Q_{N}(z)}, \quad P_{N}(z)-v(z) Q_{N}(z)=O\left(z^{N+1}\right), \quad z \rightarrow 0,
$$

где $P_{N}$ и $Q_{N}$ - многочлены порядка $N$. Следующая теорема из работы [8] утверждает сходимость (2) за пределами области, где имеет место разложение в ряд Тейлора.

ТеоремА. Для мероморфной функиии $v(z)$ и любъх $\epsilon, \delta>0$ существует такое $N_{0}$, что при $N>N_{0}$ диагональная аппроксимачия Паде (2) удовлетворяет оценке $\left|v(z)-P_{N}(z) / Q_{N}(z)\right|<\epsilon$ на всяком компактном множестве, за исключением множества меры $\delta$.

Если функция $v(z)$ является решением подходящего ОДУ, то разлагать ее в ряд Тейлора нет необходимости. Имеется эффективный алгоритм, основанный на рекуррентных непрерывных дробях. Этот алгоритм аппроксимации Паде, развитый Фейром и Льюком [9], был исходно предназначен для класса нелинейных ОДУ на вещественной оси

$$
(A+B v) v_{z z}^{\prime \prime}+(C+D v) v_{z}^{\prime}-2 B\left(v_{z}^{\prime}\right)^{2}+E+F v+G v^{2}+H v^{3}=0,
$$


где все коэффициенты $A(z), B(z), \ldots, H(z)$ имеют разложения Тейлора в некоторой точке, скажем $z=0$, и, кроме того,

$$
A(0)=B(0)=C(0)=D(0)=G(0)=H(0)=0, \quad E(0) \neq 0, \quad F(0) \neq 0 .
$$

Тогда решение можно найти в виде

$$
v(z)=\frac{\alpha_{0}}{1+\frac{\alpha_{1} z}{1+\frac{\alpha_{2} z}{1+\cdots}}}
$$

с постоянными $\alpha_{j}$, которые рекуррентно определяются из ОДУ (3) и начальных условий $v(0), v^{\prime}(0)$. Ключевая идея состоит в инвариантности уравнений (3) относительно преобразований Мёбиуса $v \mapsto(\alpha v+\beta) /(\gamma v+\delta)$.

Рекуррентная процедура строится следующим образом:

$$
v_{n}=\frac{\alpha_{n}}{1+z v_{n+1}},
$$

где $v_{n}$ и $v_{n+1}$ удовлетворяют уравнению (3) с коэффициентами $A, B, \ldots, H$, снабженными соответствующими индексами $n$ и $n+1$. Они связаны друг с другом простыми алгебраическими формулами

$$
\begin{aligned}
& A_{n+1}=-A_{n}-\alpha_{n} B_{n}, \quad B_{n+1}=-z A_{n}, \\
& C_{n+1}=-2 \frac{A_{n}+\alpha_{n} B_{n}}{z}-C_{n}-\alpha_{n} D_{n} \\
& D_{n+1}=2 A_{n}-z C_{n}, \quad F_{n+1}=-\frac{C_{n}+\alpha_{n} D_{n}}{z}+3 \frac{E_{n}}{\alpha_{n}}+2 F_{n}+\alpha_{n} G_{n}, \\
& E_{n+1}=z^{-1}\left(\alpha_{n}^{-1} E_{n}+F_{n}+\alpha_{n} G_{n}+\alpha_{n}^{2} H_{n}\right), \quad H_{n+1}=\alpha_{n}^{-1} z^{2} A_{n}, \\
& G_{n+1}=2 z^{-1} A_{n}-C_{n}+z\left(3 \alpha_{n}^{-1} E_{n}+F_{n}\right),
\end{aligned}
$$

где

$$
\alpha_{n+1}=-\frac{E_{n+1}(0)}{F_{n+1}(0)} .
$$

В работе [9] доказано, что условия (4) удовлетворяются при наличии определенных дополнительных требований на разложения Тейлора $A, B, \ldots, H$; эти условия выполнены для уравнений ПІ и ПІІ. Эти уравнения приводятся к виду (3) подстановкой

$$
u(z)=u_{0}+z u_{1}+z^{2} v(z),
$$

где $u(z)$ - решение уравнений ПІ (1) или ПІІ (см. ниже (25), (26)) с начальными условиями $u(0)=u_{0}, u^{\prime}(0)=u_{1}$. Рекурсия в уравнениях $(7)$ начинается при $n=0$, $\alpha_{0}=u_{0}, A_{0}=A(0), \ldots, H_{0}=H(0)$, и заканчивается при $v_{N+1} \equiv 0$. В результате усеченная непрерывная дробь (6) дает рациональную функцию $v_{0}$, которая является аппроксимацией Паде для $v(z)$. Сходимость алгоритма обеспечивается условиями (4), т.е. на каждом шаге все $A_{n}, B_{n}, \ldots, H_{n}$ конечны и $F_{n}(0) \neq 0[9]$.

Заметим, что численная реализация алгоритма Фейра-Льюка (6), (7) работает довольно быстро; на персональном компьютере с процессором 1.8 ГГц и оперативной памятью 2 Гбайт для получения аппроксимации 1000-го порядка (при использовании программы Mathematica 6.0) требуется 90 секунд. 


\section{3. СПЕЦИАЛЬНЫЕ РЕШЕНИЯ УРАВНЕНИЯ ПІ}

В работах [2], написанных почти столетие назад, Бутру установил основные свойства полюсов для общих решений уравнения ПІ.

1. Полюсы лежат на гладких кривых в $\mathbb{C}$, которые на бесконечности стремятся к пяти лучам $\Gamma_{k}=\{z \mid \arg z=2 \pi i k / 5, k=1,2, \ldots, 5\}$.

2. Имеется однопараметрическое семейство усеченных (tronqueé) решений, которые не имеют полюсов на бесконечности в двух последовательных секторах $\Omega_{k}=$ $\{z \mid 2 \pi i(k-1) / 5<\arg z<2 \pi i k / 5, k=1,2, \ldots, 5\}$.

3. Имеется нуль-параметрическое трижды усеченное (tritronqueé) решение, которое не имеет полюсов на бесконечности вдоль трех последовательных лучей $\Gamma_{k}$.

4. На бесконечности в секторах, где нет полюсов, эти решения имеют асимптотики

$$
u(z)= \pm \sqrt{\frac{z}{6}}\left(1+O\left(z^{-2}\right)\right), \quad z \rightarrow \infty .
$$

Позднее эти результаты были конкретизированы применительно к трансцендентам ПІ на бесконечности с использованием параметризации эллиптическими функциями (см. раздел 4). Однако распределение полюсов при $z=O(1)$ оставалось неизвестным. Несмотря на интегрируемость уравнения ПІ методами теории солитонов (см. [1], глава 5), у соответствующей задачи Римана-Гильберта нет явных решений. В отличие от высших уравнений Пенлеве, уравнение ПІ (1) не содержит свободных коэффициентов, допускающих использование преобразований Беклунда для получения явных решений из тривиального [5].

Аппроксимация Паде, описанная в разделе 2, позволяет исследовать решения уравнения ПІ при конечных z. Полюсы решения общего положения показаны на рис. 1а. Ясно видны линии полюсов Бутру, а также их асимптотическое стремление к лучам $\Gamma_{k}$ (штриховые линии).

Специальные решения уравнения ПІ (1) задаются асимптотиками

$$
u(z)= \pm \sqrt{\frac{z}{6}}+O\left(z^{-2}\right)+\frac{a}{z^{1 / 8}} \exp \left(-\frac{8}{5} \sqrt[4]{\frac{3}{2}} z^{5 / 4}\right)\left(1+O\left(z^{-3 / 8}\right)\right), \quad z \rightarrow+\infty
$$

где $a$ - произвольная постоянная [10].

Усеченное решение, показанное на рис. 1б, соответствует знаку “+" перед $\sqrt{z / 6}$ в выражении (10). Оно известно также как физическое решение "струнного уравнения" [11]

$$
u_{z z z}-12 u u_{z}+1=0 .
$$

Численно это решение неустойчиво; почти любое начальное условие $u(0), u^{\prime}(0)$ дает $u(z) \sim-\sqrt{z / 6}, z \rightarrow+\infty$, или сингулярное решение с полюсом в $z \in \mathbb{R}^{+}$. Правильные начальные условия для усеченных решений определяется из граничной задачи для уравнения (1) с граничными условиями

$$
u(0)=u_{0}, \quad u(L) \sim+\sqrt{\frac{L}{6}}, \quad L \gg 1,
$$



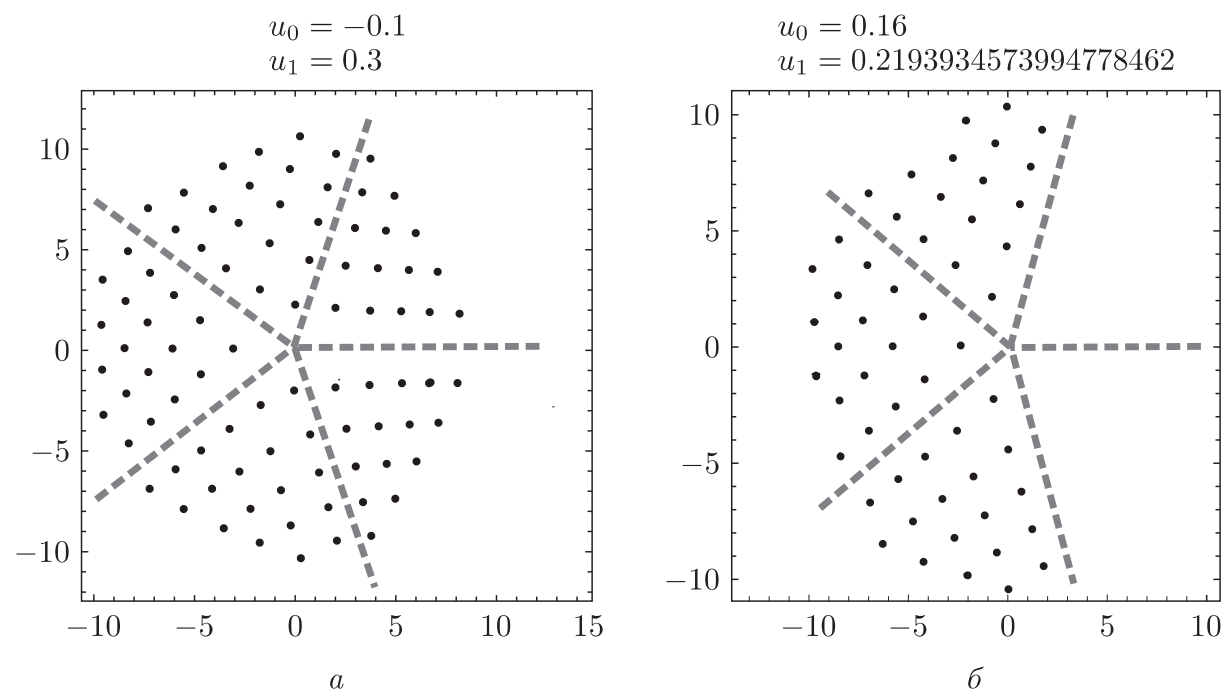

Рис. 1. Общее решение (а) и усеченное решение (б). Штриховые линии лучи $\Gamma_{k}$.

Граничная задача (1), (11) имеет решение для любого $u_{0}$, что дает необходимое значение $u^{\prime}(0)$. Заметим, что здесь требуется достаточно высокая точность вычислений, иначе в двух правых секторах на рис. 16 появляются дуги “лишних" полюсов.

Другое семейство специальных решений задается асимптотическим разложением (10) со знаком “-” перед $\sqrt{z / 6}$. Распределение полюсов для этого однопараметрического семейства показано на рис. 2б. Этот класс содержит трижды усеченное решение, впервые найденное Бутру [2], а в настоящее время используемое в ряде физических приложений (см. [11]-[13]). Оно соответствует значению $a=0$ в асимптотике (10). Начальные условия $u(0), u^{\prime}(0)$ нельзя найти явно с использованием соответствующей изомонодромной техники (см. раздел 4), и поэтому они оцениваются численно исходя из граничной задачи на мнимой оси $z \in i \mathbb{R}$ для уравнения (1) с граничными условиями

$$
u(-i L)=-\sqrt{\frac{-i L}{6}}, \quad u(i L)=-\sqrt{\frac{i L}{6}}, \quad L \gg 1 .
$$

Это дает значения

$$
u(0)=-0.1875543083975161 \ldots, \quad u^{\prime}(0)=0.3049055603911820 \ldots,
$$

которые совпадают с результатами работ [12] и [14]. Численно контролировать экспоненциально малые члены в (10) нелегко, и поэтому граничную задачу (12) необходимо решать с максимально возможной точностью, чтобы обеспечить $a=0$ в (10). На рис. 2 представлены полюсы трижды усеченного решения, вычисленные при начальных условиях $u(0), u^{\prime}(0)$, которые аппроксимируют истинные значения 


$$
\begin{array}{ll}
u_{0}=-0.1875543083411885265172225324883521313 & u_{0}=-0.1875543083411885 \\
u_{1}=0.3049055602602021202437399456075686951 & u_{1}=0.3049055602602021
\end{array}
$$
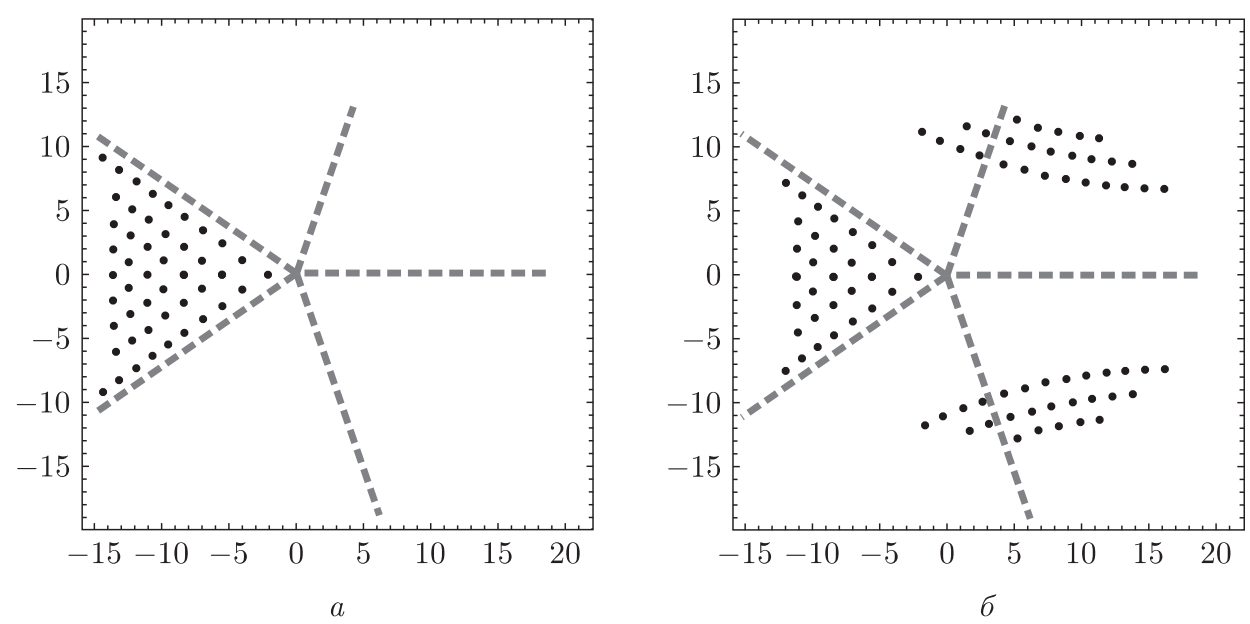

Рис. 2. Полюсы трижды усеченных решений с точностью начальных условий 64 (а) и 16 (б) десятичных знаков.

с точностью 64 и 16 десятичных знаков. При увеличении точности в начальных условиях "лишние" дуги полюсов удаляются от начала координат. В действительности на рис. 2 "лишние" полюсы присутствуют в обоих случаях, но на рис. 2а они находятся вне отображаемой области. Первый полюс (ближайший к началу координат) трижды усеченного решения был найден в работах [14], [12] другим численным способом; в результате получилось значение $z_{0}=-2.3841688$. Указанная выше аппроксимация Паде порядка 200 дает следующие значения первых десяти полюсов:

$$
\begin{aligned}
z_{0} & =-2.384168769564550 \\
z_{1,2} & =-4.071055433084488 \pm 1.3355514706611683 i \\
z_{3} & =-5.664449429020133 \\
z_{4,5} & =-5.573539567139687 \pm 2.489136512145025 i \\
z_{6,7} & =-6.971620941710831 \pm 3.548265719706162 i \\
z_{8,9} & =-7.110821873372145 \pm 1.162265719858028 i
\end{aligned}
$$

Эти численные результаты подтверждают следующую гипотезу Дубровина.

ПрЕДЛОЖЕНИЕ 1. Если решение уравнения ПІ не имеет полюсов на бесконечности в секторе $\Omega_{k}$, то оно не имеет полюсов во всем секторе $\Omega_{k}$.

Это предположение впервые было сформулировано в работе [12], где исследование волнового коллапса в нелинейном уравнении Шредингера потребовало использования трижды усеченного решения как автомодельной моды в момент градиентной катастрофы. Численное моделирование этого решения, выполненное в работе [12], согласуется с рис. 2 и со значениями (13). 
Аналитическое доказательство этой гипотезы будет опубликовано автором в отдельной статье. Оно основано на методе изомонодромных деформаций [1] и асимптотическом исследовании соответствующей задачи Римана-Гильберта.

\section{4. ПАРАМЕТРИЗАЦИЯ РЕШЕНИЙ УРАВНЕНИЯ ПІ, АСИМПТОТИКИ НА БЕСКОНЕЧНОСТИ}

Уравнение (1) интегрируется с помощью метода изомонодромных деформаций ([1], глава 5). Условие совместности уравнений пары Лакса для $(2 \times 2)$-матричной функции $\Psi=\Psi(\lambda, z)$

$$
\begin{aligned}
& \Psi_{\lambda}=\left(\begin{array}{cc}
u_{z} & 2 \lambda^{2}+2 u \lambda-z+2 u^{2} \\
2(\lambda-u) & -u_{z}
\end{array}\right) \Psi \\
& \Psi_{z}=-\left(\begin{array}{cc}
0 & \lambda+2 u \\
1 & 0
\end{array}\right) \Psi
\end{aligned}
$$

эквивалентно уравнению (1). Канонические матричные решения $\Psi_{k}(\lambda, z)$ системы (14) однозначно определяются своим асимптотическим поведением

$$
\begin{aligned}
\Psi_{k}(\lambda, z) \sim & \frac{1}{\sqrt{2}}\left(\begin{array}{cc}
\lambda^{1 / 4} & \lambda^{1 / 4} \\
\lambda^{-1 / 4} & -\lambda^{-1 / 4}
\end{array}\right)\left[1-\frac{1}{\sqrt{\lambda}}\left(\begin{array}{cc}
H & 0 \\
0 & -H
\end{array}\right)+\right. \\
& \left.+\frac{1}{2 \lambda}\left(\begin{array}{cc}
H^{2} & u \\
u & H^{2}
\end{array}\right)+O\left(\lambda^{-3 / 2}\right)\right] e^{\theta(\lambda, z) \sigma_{3}}, \quad|\lambda| \rightarrow \infty, \quad \lambda \in \Sigma_{k},
\end{aligned}
$$

в секторах

$$
\Sigma_{k}=\left\{\lambda \in \mathbb{C} \mid \frac{2 \pi}{5}\left(k-\frac{3}{2}\right)<\arg \lambda<\frac{2 \pi}{5}\left(k+\frac{1}{2}\right)\right\}, \quad k \in \mathbb{Z},
$$

где

$$
\theta(\lambda, z)=\frac{4}{5} \lambda^{5 / 2}-z \lambda^{1 / 2}, \quad \sigma_{3}=\left(\begin{array}{cc}
1 & 0 \\
0 & -1
\end{array}\right), \quad H=\frac{1}{2} u_{z}^{2}-2 u^{3}+z u
$$

Разрез на комплексной $\lambda$-плоскости для случая дробных степеней $\lambda$ выбран вдоль отрицательной вещественной полуоси.

Матрицы Стокса $S_{k}$ определяются из условий сопряжения

$$
\Psi_{k+1}(\lambda, z)=\Psi_{k}(\lambda, z) S_{k}, \quad \lambda \in \Sigma_{k} \cap \Sigma_{k+1} .
$$

Они имеют треугольный вид

$$
S_{2 k-1}=\left(\begin{array}{cc}
1 & s_{2 k-1} \\
0 & 1
\end{array}\right), \quad S_{2 k}=\left(\begin{array}{cc}
1 & 0 \\
s_{2 k} & 1
\end{array}\right)
$$

и удовлетворяют соотношениям

$$
S_{k+5}=\sigma_{1} S_{k} \sigma_{1}, \quad S_{1} S_{2} S_{3} S_{4} S_{5}=i \sigma_{1}, \quad \sigma_{1}=\left(\begin{array}{ll}
0 & 1 \\
1 & 0
\end{array}\right) .
$$


В силу (19) два множителя Cтокса $s_{k}$ определяют все остальные; они не зависят ни от $\lambda$, ни от $z$, если $u(z)$ удовлетворяет уравнению (1). Поскольку все $\Psi_{k}$ являются аналитическими при $\lambda \in \Sigma_{k}$, условия сопряжения (17) вместе с нормировкой (15) определяют задачу Римана-Гильберта в секторах $\Sigma_{k}$. Eе решение $\Psi_{k}(\lambda, z)$ дает решение $u(z)$ уравнения ПІ (1) с помощью формулы обращения

$$
u(z)=\sqrt{2} \lim _{\lambda \rightarrow \infty}\left\{\left(\sigma_{3}+\sigma_{1}\right) \lambda^{1-\sigma_{3} / 4} \Psi_{k}(\lambda, z) e^{-\theta(\lambda, z) \sigma_{3}}\right\}_{21} .
$$

Данные Стокса $\left\{s_{k}\right\}$ параметризуют специальные решения уравнения (1) следующим образом:

$$
\begin{array}{lll}
s_{1}=0, & s_{4}=s_{3}=s_{2}+s_{5}=i & \\
s_{1}=s_{5}=0, & s_{4}=s_{3}=s_{2}=i & \text { - тсеченные решения, }
\end{array}
$$

Свободный параметр $s_{1}$ в $(21)$ связан с асимптотическим коэффициентом $a$ в $(10)$ условием [10]

$$
a=\frac{s_{1}}{2^{11 / 8} 3^{1 / 8} \sqrt{\pi}} .
$$

Задача Римана-Гильберта (15), (17), (18) при специальном выборе параметров (21) имеет единственное решение при любом $z \in \mathbb{C}$, не совпадающем с полюсом функции $u(z)$ (см. [10]). $\mathrm{K}$ сожалению, для произвольных ненулевых $\left\{s_{1}, \ldots, s_{5}\right\}$ явных решений нет; даже при $z=0$ начальные условия $u(0)$ и $u^{\prime}(0)$ нельзя найти явно.

Однако распределение полюсов при $|z| \rightarrow \infty$ можно найти из сформулированной выше задачи Римана-Гильберта. Главный член дается представлением $u(z)$ в терминах эллиптической функции [13]:

$$
\begin{aligned}
u(z)= & -e^{\pi i / 10} \sqrt{z} \wp\left(\frac{4}{5} e^{-i \varphi / 5+2 \pi i / 5} z^{5 / 4}+\chi(\varphi) ; g_{2}(\varphi), g_{3}(\varphi)\right) \times \\
& \times\left(1+O\left(z^{-1}\right)\right), \quad|z| \rightarrow \infty, \quad \varphi=\arg z, \quad \frac{4 \pi}{5}<\varphi<\frac{6 \pi}{5},
\end{aligned}
$$

где $\wp=\wp\left(z ; g_{2}, g_{3}\right)$ - эллиптическая функция Вейерштрасса, $\left(\wp^{\prime}\right)^{2}=4 \wp^{3}-g_{2} \wp-g_{3}$, с базисом полупериодов

$$
\omega_{1,3}=\int_{\infty}^{\lambda_{1}, \lambda_{3}}\left(4 \lambda^{3}-g_{2} \lambda-g_{3}\right)^{-1 / 2} d \lambda .
$$

Эллиптическая кривая

$$
w^{2}(\lambda)=4 \lambda^{3}-g_{2} \lambda-g_{3} \equiv 4\left(\lambda-\lambda_{1}\right)\left(\lambda-\lambda_{2}\right)\left(\lambda-\lambda_{3}\right)
$$

деформирована по параметру $\varphi$ с помощью решения следующей задачи Бутру:

$$
\begin{gathered}
\lambda_{1}+\lambda_{2}+\lambda_{3}=0 \\
\lambda_{1} \lambda_{2}+\lambda_{2} \lambda_{3}+\lambda_{3} \lambda_{1}=2 e^{i \varphi}, \\
\operatorname{Re} \int_{\lambda_{1}}^{\lambda_{2}} w(\lambda) d \lambda=0, \quad \operatorname{Re} \int_{\lambda_{1}}^{\lambda_{3}} w(\lambda) d \lambda=0,
\end{gathered}
$$




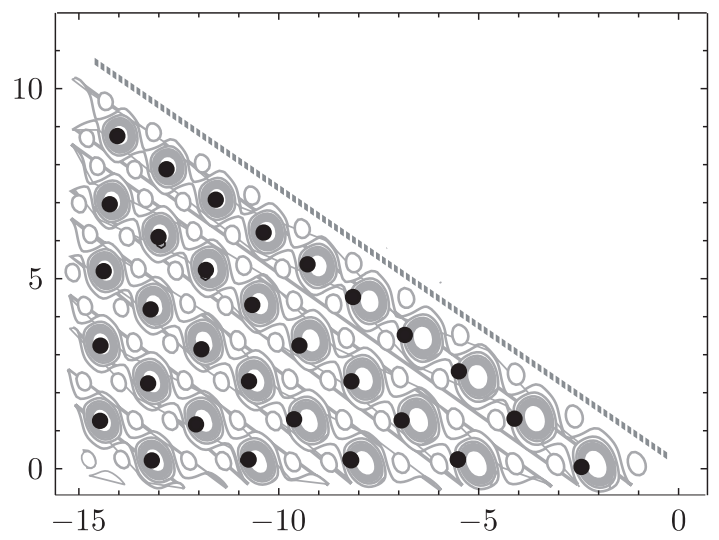

Рис. 3. Полюсы трижды усеченных решений (черные точки) в сравнении с полюсами функции Вейерштрасса (22) (серые концентрические "окружности").

с граничными условиями

$$
\begin{aligned}
& \lambda_{1,3} \rightarrow-\frac{1}{\sqrt{6}} \quad \text { при } \quad \varphi \rightarrow \frac{4 \pi}{5}, \\
& \lambda_{2,3} \rightarrow \frac{1}{\sqrt{6}} e^{-\pi i / 5} \quad \text { при } \quad \varphi \rightarrow \frac{6 \pi}{5} .
\end{aligned}
$$

Из функциональных уравнений (23), (24) следуют выражения для модулей эллиптической функции (22):

$$
g_{2}(\varphi)=-2 e^{i \varphi-\pi i / 5}, \quad g_{3}(\varphi)=4 \lambda_{1}(\varphi) \lambda_{2}(\varphi) \lambda_{3}(\varphi)
$$

Фазовый сдвиг в (22) дается формулой [13]

$$
\chi(\varphi)=-\frac{\omega_{1}(\varphi)-\omega_{3}(\varphi)}{\pi i} \ln \left(i s_{4}\right)-\frac{\omega_{3}(\varphi)}{\pi i} \ln \left(i s_{3}\right)+O\left(z^{-1}\right), \quad s_{3} s_{4} \neq 0,
$$

где $s_{3}, s_{4}$ - параметры Стокса задачи Римана-Гильберта (15), (17), (18).

Большое число полюсов, полученных из аппроксимаций Паде в разделе 3 , позволяет сравнить их распределение на бесконечности с явными асимптотиками (22). Один из результатов показан на рис. 3 , где трижды усеченное решение $\left(s_{3}=s_{4}=i\right)$ изображено в секторе $\pi<\arg z<4 \pi / 5$. Черные точки обозначают полюсы, найденные из аппроксимации Паде (5) (ср. рис. 2а). Серые концентрические "окружности" являются проекциями линий уровня больших абсолютных значений функции Вейерштрасса (22). Результаты численного счета показывают, что хорошее совпадение достигается при довольно малых значениях $|z| \approx 15$. Другие решения уравнения ПІ демонстрируют аналогичный масштаб совпадений с асимптотиками (22). 


\section{5. СПЕЦИАЛЬНЫЕ РЕШЕНИЯ УРАВНЕНИЯ ПІІ}

Рассмотренная в разделе 2 аппроксимация Паде применима также и для уравнения ПІІ. Следующие примеры иллюстрируют распределение полюсов двух хорошо известных решений.

1. Решение Трейси-Уидома используется в ряде приложений, таких как теория случайных матриц и случайных перестановок [15], в качестве основного компонента функции распределения собственных значений $(n \times n)$-матриц и длин крюков диаграмм Юнга при $n \rightarrow \infty$. Оно удовлетворяет уравнению ПІІ

$$
u_{z z}=z u+2 u^{3}, \quad z \in \mathbb{R},
$$

с асимптотиками на бесконечности

$$
\begin{array}{ll}
u(z) \sim \frac{1}{2 \sqrt{\pi}} z^{-1 / 4} \exp \left(-\frac{2}{3} z^{3 / 2}\right), & z \rightarrow+\infty, \\
u(z) \sim \sqrt{-\frac{z}{2}}\left(1+\frac{1}{9 z^{5}}-\frac{73}{128 z^{6}}+\cdots\right), & z \rightarrow-\infty .
\end{array}
$$

Как показано на рис. 4, все полюсы находятся вдали от вещественной оси; именно поэтому решение не осциллирует на вещественной оси.
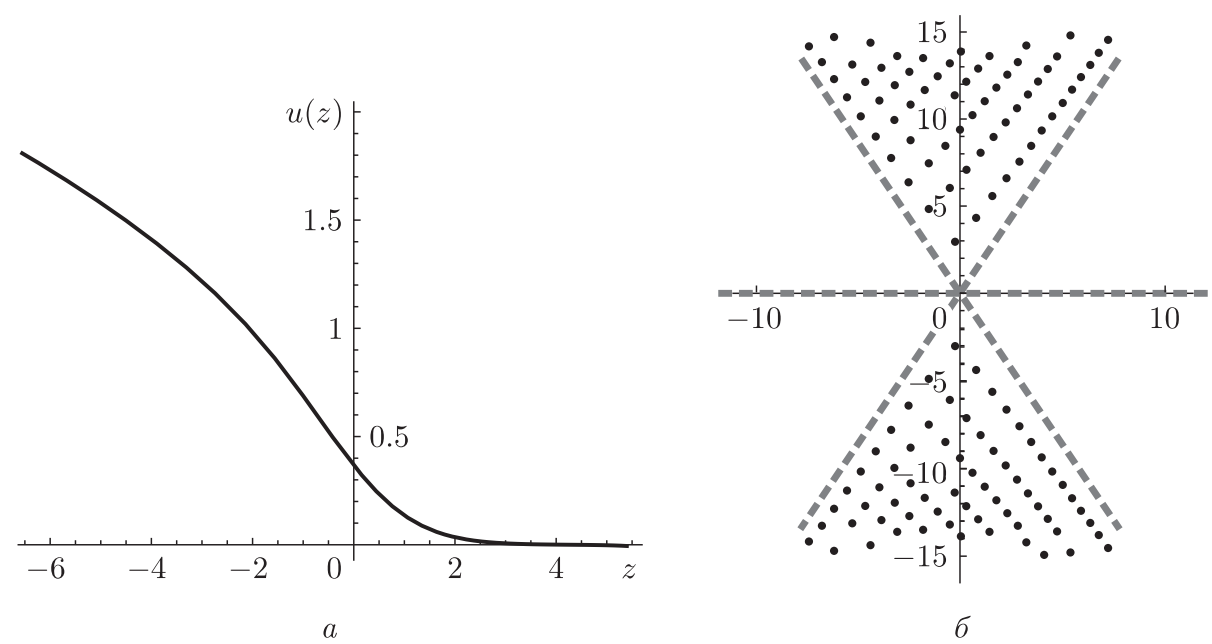

Рис. 4. Решение Хастингса-Маклеода (а) и его полюсы в комплексной плоскости (б).

Решение Хастингса-Маклеода можно рассматривать как усеченное решение уравнения ПІІ (25). Лучи $\arg z=\pi k / 3, k=1,2, \ldots, 6$, играют роль лучей $\Gamma_{k}$ для уравнения ПІ, т.е. все линии полюсов на бесконечности стремятся к этим лучам. Численное исследование методом Паде-аппроксимации показывает, что в двух парах последовательных секторов полюсы отсутствуют (см. рис. 4). Таким образом, имеет место следующее предположение, аналогичное предложению 1. 


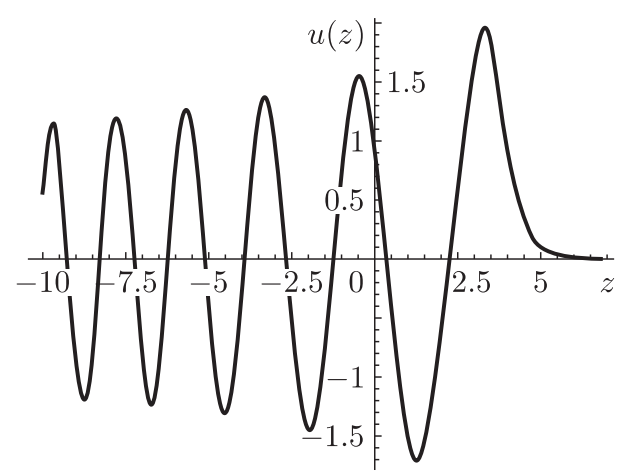

$a$

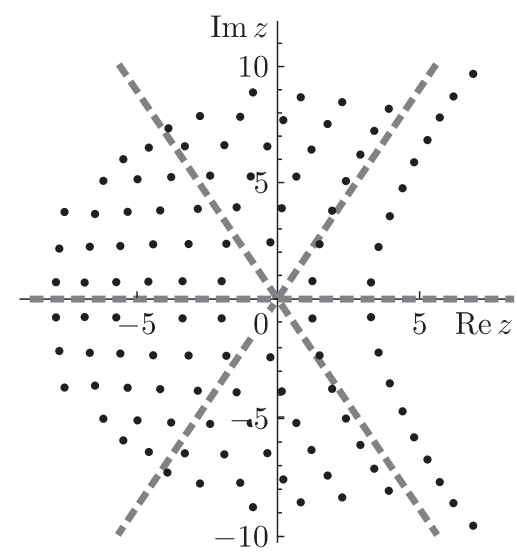

б

Рис. 5. Решение Абловица-Сегура (а) и его полюсы (б).

ПреДЛОЖениЕ 2. Решение Трейси-Уидома уравнения ПІІ (25) не имеет полюсов в секторах $|\arg z|<\pi / 3 u 2 \pi / 3<\arg z<4 \pi / 3$.

Асимптотические разложения на бесконечности в терминах эллиптических функций были найдены в монографии [1] (см. главы 7, 8). Было установлено отсутствие полюсов в двух парах секторов на бесконечности. Однако полное доказательство предложения 2 отсутствует и является предметом последующей публикации.

2. Решение Абловица-Сегура используется как сшивка между осциллирующими и обращающимися в нуль решениями модифицированного уравнения Кортевега-де Фриза. Оно удовлетворяет другому варианту уравнения ПІІ:

$$
u_{z z}=z u-2 u^{3}
$$

с асимптотиками

$$
\begin{array}{ll}
u(z) \sim \frac{a}{2 \sqrt{\pi}} z^{-1 / 4} \exp \left(-\frac{2}{3} z^{3 / 2}\right), & z \rightarrow+\infty, \\
u(z) \sim b(-z)^{-1 / 4} \sin \left(\frac{2}{3}(-z)^{3 / 2}-\frac{3}{4} b^{2} \ln (-z)+\theta\right), & z \rightarrow-\infty .
\end{array}
$$

Две последовательности полюсов вдоль отрицательной вещественной полуоси вызывают колебания, тогда как отсутствие полюсов вокруг положительной полуоси приводит к неосциллирующей моде (см. рис. 5). Внешняя правая линия полюсов играет здесь роль границы множества полюсов. Таким образом, предложение, аналогичное предложениям 1 и 2, должно быть сформулировано относительно этой линии вместо границ критических секторов.

Благодарности. Работа частично поддержана РФФИ (гранты № 07-01-00081, 06-01-95052). 


\section{Список литературы}

[1] A. S. Fokas, A. R. Its, A. A. Kapaev, V. Yu. Novokshenov, Painlevé Transcendents. The Riemann-Hilbert Approach, Math. Surveys Monogr., 128, AMS, Providence, RI, 2006.

[2] P. Boutroux, Ann. École Norm., 30 (1913), 255-375; Ann. École Norm., 31 (1914), 99-159.

[3] А. И. Яблонский, Вести АН БССР. Сер. физ.-тех. наук, 3 (1959), 30-35; А. П. Воробьев, Дифферени. уравнения, 1 (1965), 58-59.

[4] K. Okamoto, Math. Ann., 275:2 (1986), 221-255.

[5] P. A. Clarkson, Phys. Lett. A, 319:1-2 (2003), 137-144; J. Math. Phys., 44:11 (2003), 5350-5374; Eur. J. Appl. Math., 17:3 (2006), 293-322.

[6] В. Г. Марихин, А. Б. Шабат, М. Бойти, Ф. Пемпинелли, ЖЭТФ, 117:3 (2000), 634-643.

[7] М. Х. Чанкаев, А. Б. Шабат, ТМФ, 157:2 (2008), 175-187.

[8] J. Nuttall, J. Math. Anal. Appl., 31:1 (1970), 147-153.

[9] W. Fair, Y.L. Luke, Math. Comp., 20:96 (1966), 602-606.

[10] A. A. Kapaev, J. Phys. A, 37:46 (2004), 11149-11167.

[11] П. Г. Гриневич, С. П. Новиков, Алгебра и анализ, 6:3 (1994), 118-140.

[12] B. Dubrovin, T. Grava, C. Klein, Nonlinear Sci., 19:1 (2009), 67-94; arXiv: 0704.0501.

[13] А. А. Капаев, Дифферени. уравнения, 24:10 (1988), 1684-1695.

[14] N. Joshi, A. V. Kitaev, Stud. Appl. Math., 107:3 (2001), 253-291.

[15] C. Tracy, H. Widom, Comm. Math. Phys., 177:3 (1996), 727-754. 